\title{
Dispersion Activity
}

National Cancer Institute

\section{Source}

National Cancer Institute. Dispersion Activity. NCI Thesaurus. Code C150035.

Transformation of a solid or gas into a dispersion or suspension by mixing it with a specified liquid. 